\title{
Do locally defined feature discontinuities capture attention?
}

\author{
CHARLES L. FOLK and SUSAN ANNETT \\ Villanova University, Villanova, Pennsylvania
}

\begin{abstract}
Results from previous visual search studies have suggested that abrupt onsets produce involuntary shifts of attention (i.e., attentional capture), but discontinuities in simple features such as color and brightness do not (Jonides \& Yantis, 1988). In the present study we tested whether feature discontinuities (i.e., "singletons") can produce attentional capture in a visual search task if defined "locally" or over a small spatial range. On each trial, a variable number of letters appeared, one of which differed from the others in color or intensity. The location of this singleton was uncorrelated with target location. Local discontinuities were created by embedding the letters in a dot texture. In Experiment 1, display size effects for singleton targets were not reduced with the addition of a background dot texture. Similar results were obtained in Experiment 2, regardless of variations in texture density. Experiment 3 confirmed that when targets are defined by a color or intensity singleton, they are detected preattentively, and that increasing texture density yields faster detection. We conclude that the spatial range over which feature discontinuities are defined may influence the guidance of spatial attention, but it has no influence on their ability to capture attention.
\end{abstract}

The visual search task is an invaluable tool in research on perception and cognition. In the most general terms, the task involves searching a visual display for a prespecified target. Changes in task performance resulting from systematic variations in the characteristics of the target and distractors can inform and distinguish between models of visual information processing - in particular, theories of visual selective attention (e.g., Duncan \& Humphreys, 1989; Egeth, Jonides, \& Wall, 1972; Treisman \& Gelade, 1980; Wolfe, Cave, \& Franzel, 1989).

In an important series of studies, Yantis and his colleagues (Jonides \& Yantis, 1988; Yantis \& Johnson, 1990; Yantis \& Jones, 1991; Yantis \& Jonides, 1984, 1990) demonstrated that performance on a visual search task can be strongly influenced by the temporal characteristics of the display elements. Specifically, when a target element is abruptly onset among a variable number of gradually onset distractors (presented by removing camouflaging line segments), response time does not vary with the number of distractors. This pattern obtains even when a distractor is just as likely as the target to be the abruptly onset element, suggesting that abrupt onsets

This research was supported in part by National Institute of Mental Health Grant 1 RO3 MH 45008 to C.L.F. Experiment 1 was presented at the meeting of the Eastern Psychological Association, March 1990. Experiment 2 was based on the master's thesis of the second author, submitted to Villanova University. We would like to thank William Bacon, Howard Egeth, John Jonides, Arthur Kramer, Jan Theeuwes, and Steven Yantis for helpful comments regarding this work, and Nathalie Menedes for help with running subjects. Correspondence should be addressed to C. L. Folk, Department of Psychology, Villanova University, Villanova, PA 19085 (e-mail: chipfolk@villvm. bitnet). can "capture" visual attention by eliciting an involuntary shift of attentional resources.

Jonides and Yantis (1988, Experiments 1 and 2) investigated the possibility that spatial attention is not captured by abrupt onset per se, but by any display element that is "unique" with respect to some feature. They compared conditions in which an abruptly onset element appeared among no-onset elements with conditions in which a "singleton" in color or intensity appeared among an otherwise homogeneous group of elements. Search performance was enhanced (i.e., response time did not increase with display size) only when the target was characterized by an abrupt onset. The authors concluded that attentional capture is specific to abrupt visual onset and is not elicited by feature discontinuities, or singletons. ${ }^{1}$

Theeuwes (1990) reached a similar conclusion on the basis of studies involving discontinuities in color and form. Subjects searched for a horizontal line segment inside 1 of 4,8 , or 16 enclosing objects. On each trial, 1 object was a singleton in shape or color. The location of the singleton was uncorrelated with target location. Consistent with the results of Jonides and Yantis (1988), search rates were unaffected by whether or not the target appeared inside the singleton object, providing no evidence of attentional capture.

The fact that feature discontinuities did not yield evidence of attentional capture in these studies is somewhat surprising. Color, brightness, and form are members of a set of "simple" visual features that are processed preattentively and produce "pop-out" when specified as the target in a visual search task (e.g., Connor, 1972; Treisman \& Gelade, 1980; Wolfe et al., 1989). Many authors have argued that the preattentive 
detection of discontinuities in such "simple" features serves as the basis by which focal attention is attracted to particular regions of the visual array (e.g., Bergen \& Julesz, 1983; Cave \& Wolfe, 1990; Duncan \& Humphreys, 1989; Folk \& Egeth, 1989; Kahneman \& Henik, 1981; Koch \& Ullman, 1985; Neisser, 1967; Sagi \& Julesz, 1987; Treisman \& Souther, 1985; Wolfe et al., 1989). Moreover, recent studies have shown that irrelevant singletons in color and form can produce significant distraction effects when searching for other feature singletons (Bacon \& Egeth, 1994; Folk, Remington, \& Johnston, 1992; Folk, Remington, \& Wright, 1994; Pashler, 1988, Experiment 6; Theeuwes, 1991, 1992).

Why, then, was there no evidence that attention was attracted to discontinuities in color or brightness in Jonides and Yantis's (1988) study? One possibility, as suggested by the authors, is that such discontinuities play a role in guiding focal attention (see Wolfe et al., 1989), but they do not automatically attract attention in the sense of eliciting involuntary, reflexive shifts of attention. In support of this conclusion, Jonides and Yantis (1988, Experiment 3 ) found that when the feature discontinuity was completely correlated with target location, search performance was enhanced.

Another possibility, however, is that the displays used by Jonides and Yantis (1988) did not provide an adequate test of the ability of feature discontinuities to capture attention. Many models of preattentive processing assume that discontinuity detection is a local process involving the comparison of features across a relatively restricted spatial area (e.g., Julesz, 1986; Koch \& Ullman, 1985; Sagi \& Julesz, 1987; Ullman, 1984; Wolfe, 1992). Koch and Ullman, for example, suggest that feature discontinuities are coded through lateral inhibition among neighboring feature detectors; a display element that shares features with its neighbors is inhibited and thus less likely to attract attention than an element that differs from its neighbors. Julesz proposed a similar idea in terms of locally tuned detectors responsive to "feature gradients."

Behavioral support for a local effect in feature discontinuity detection can be found in work on texture segregation as well as visual search (Bacon \& Egeth, 1991; Beck, Prazdny, \& Rosenfeld, 1983; Bergen \& Julesz, 1983; Bravo \& Nakayama, 1992; Nothdurft, 1985, 1992; Sagi \& Julesz, 1987). For example, Nothdurft $(1985,1992)$ has shown that texture segregation based on discontinuities in simple features is enhanced as the spacing between texture elements decreases. Similarly, Sagi and Julesz found that the detection of a single oblique line segment in a field of orthogonal line segments is enhanced as interelement distance decreases to $2^{\circ}$ of visual angle or less. Bravo and Nakayama reported similar results under conditions in which the target and distractor features were unknown. Finally, Bacon and Egeth (1991) confirmed that interelement spacing influences the detection of feature discontinuities in visual search, but found that the effect is specific to the proximity of nontargets.
In the study by Jonides and Yantis (1988), stimulus elements were presented at relatively large interelement distances; they appeared at the vertices of an imaginary hexagon with sides that were $5.7^{\circ}$ of visual angle. At these distances, the feature singletons may not have triggered the hypothesized local discontinuity detectors. Assuming that attentional capture is dependent on the activation of such detectors, the "globally" defined singletons used by Jonides and Yantis would not be expected to produce capture. ${ }^{2}$ Theeuwes $(1990)$, on the other hand, used displays with much smaller interelement distances (approximately $2^{\circ}$ ) and still found no evidence of attentional capture by color or form singletons. The possibility remains, however, that discontinuities defined over ranges of less than $2^{\circ}$ can produce capture.

In the present studies, the spatial range over which feature singletons are defined was systematically manipulated to provide a direct test of the effects of this variable on the ability of feature singletons to capture attention. One means of manipulating spatial range is to systematically vary the distances between the to-besearched display elements. For a given number of display elements, however, such a manipulation necessarily changes the geometry of the search set and thus may impact processes other than those of interest. Consequently, we chose to manipulate spatial range by embedding display characters in background textures of varying density, enabling a comparison of range effects for otherwise identical displays.

\section{EXPERIMENT 1}

The first experiment was patterned after the color and intensity conditions from Experiment 1 of Jonides and Yantis (1988). Two or four letters appeared on each trial. One of the letters was a singleton in color or intensity, and its location was uncorrelated with the target location. In one condition, the letters were embedded in a texture consisting of small dots that were the same color and brightness as the nonsingleton characters. The dots were separated from the search letters and each other by approximately $1^{\circ}$ of visual angle. Performance in this texture condition was compared with that of a condition in which no background texture elements appeared (similar to the displays used by Jonides \& Yantis, 1988). If the range over which spatial discontinuities are defined influences their ability to capture attention, then attentional capture should be evident in the texture condition, but not in the no-texture condition. Specifically, in the texture condition, there should be no effect of display size when the target happens to be the singleton.

\section{Method}

Subjects. Thirty-two Villanova undergraduates participated in a single 1-h session in partial fulfillment of a course requirement. All the subjects reported normal or corrected-to-normal visual acuity and normal color vision. Sixteen subjects were randomly assigned to the color condition, and 16 were assigned to the intensity condition. For half of the subjects in the color condition, 
the discontinuity was a red letter among green; for the other half, it was a green letter among red.

Apparatus. Stimulus displays were presented in a small, dimly lit room on a Princeton Graphics SR-12 monitor. This was driven by a Zenith 286 microcomputer equipped with a Sigma Design, Color 400 high-resolution $(680 \times 400)$ graphics board. The monitor was placed at eye level on a wooden stand at a viewing distance of approximately $50 \mathrm{~cm}$. The subjects responded by pressing keys on the computer keyboard, which was centered on the table in front of them.

Stimuli. The letters appearing on any given trial were randomly chosen without replacement from the set E, H, P, S, and U. From a viewing distance of approximately $50 \mathrm{~cm}$, the letters subtended $.5^{\circ}$ of visual angle in width and $.6^{\circ}$ in height, and they were created by illuminating segments of a block figure eight (see Jonides \& Yantis, 1988). The letters appeared $3.7^{\circ}$ from fixation, at the vertices of an imaginary octagon. When four letters were presented, they appeared in either a "square" or "diamond" configuration (determined randomly on each trial). When two letters were presented, they appeared at diametrically opposed locations (again determined randomly on each trial).

In the no-texture condition, the letters appeared alone against the black CRT screen. In the texture condition, the letters were embedded in a texture of small dots, each measuring $.23^{\circ} \times .23^{\circ}$ of visual angle (see Figure 1). The dots were arranged in a $9 \times 9$ matrix and were separated from one another by $.92^{\circ}$. The entire matrix subtended $10.9^{\circ} \times 10.9^{\circ}$ of visual angle. Texture dots were removed from anywhere a letter occurred.

Design. A mixed design with one between-subjects factor and three within-subjects factors was used. The between-subjects factor was discontinuity type (color or intensity). Following Jonides and Yantis (1988), every trial in each of these conditions contained exactly one singleton letter (i.e., a letter different from the rest in color or intensity). For half of the subjects in the color condition the singleton letter was red (IBM computer graphics card, Color Designation 12), and the remaining display elements (including texture dots) were green (IBM Color 10). The remaining subjects received the reverse color assignment. In the intensity condition, the singleton was bright white (IBM Color 15; approximately $40 \mathrm{~cd} / \mathrm{m}^{2}$ ) and the remaining display elements were medium white (IBM Color 7; approximately $10 \mathrm{~cd} / \mathrm{m}^{2}$ ).

The three within-subjects factors were background (no-texture, texture), display size (two, four letters), and trial type (present-singleton, present-nonsingleton, absent). Each subject received one practice block followed by three experimental blocks of 80 trials in each of the two background conditions. The order of background conditions was balanced across subjects. Half of the trials in each block were Display Size 2, and half were Display Size 4. On half of the trials a target was present, and on half it was absent. When a target was present, it was the singleton exactly $1 / n$th of the time, where $n$ is display size. Thus, the location of the target was completely uncorrelated with the location of the singleton letter. The order of trial types and display size was randomly determined within each block.

Procedure. The subjects received written and oral descriptions of the experimental task. They were explicitly informed of the irrelevant nature of the feature singleton and were told that their best strategy would be to ignore the feature difference. At the start of each block of trials, instructions describing the upcoming background condition and block number appeared on the screen. The subjects pressed the "enter" button on the numeric keypad of the keyboard to initiate a block of trials.

For all conditions, each trial began with a $1,000-\mathrm{msec}$ presentation of a white target letter in the center of an otherwise blank screen. The stimulus display then appeared and remained on the screen until the subject responded, at which time the screen turned blank. The target for the next trial appeared after a $1,500-\mathrm{msec}$ intertrial interval (ITI). The subjects were instructed to press a key
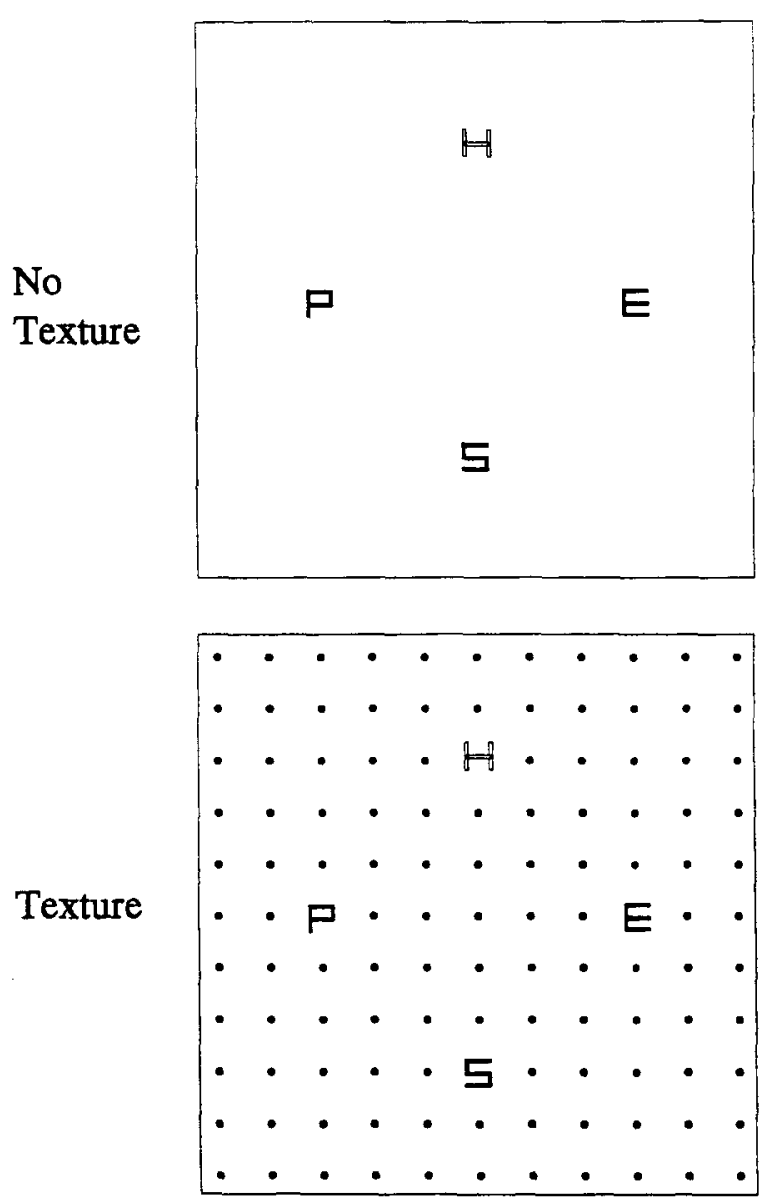

Figure 1. Examples of four-character displays in the no-texture and texture conditions.

labeled "present" with the index finger of their dominant hand if the target was present, and a key labeled "absent" with the index finger of their nondominant hand if the target was not present. The " 0 " and "." keys on the numeric keypad of the computer keyboard were used as response keys.

Response time was the primary dependent variable, and it was measured by the computer from the onset of the stimulus display until a key was depressed. Response times less than $200 \mathrm{msec}$ and greater than $5,000 \mathrm{msec}$ were scored as errors. Error trials were followed by a $1000-\mathrm{Hz}$ tone for $500 \mathrm{msec}$ and a "buffer" trial, whose parameters were randomly chosen from the set for that block. Response times from error trials and buffer trials were not included in the data analysis.

\section{Results}

Mean response times for the experimental trials in the color and intensity conditions are plotted in Figure 2, and error rates are listed in Table 1. Means for the color condition are collapsed across color assignment, since the data analyses showed no main effect or interactions associated with this variable.

Separate analyses of variance (ANOVAs) were performed on the color and intensity response times. The factors included background (texture, no-texture), trial type (present-singleton, present-nonsingleton, absent), 
COLOR

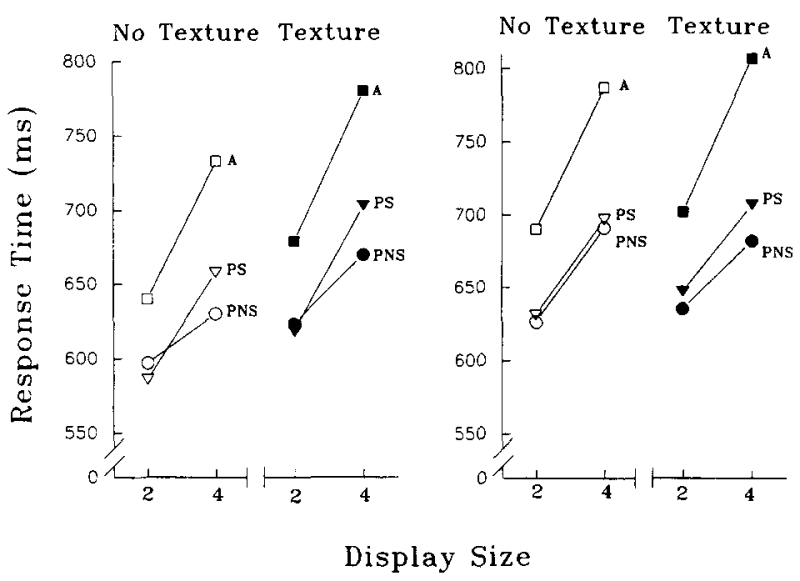

Figure 2. Average mean response times as a function of trial type (A, absent; PS, present-singleton; PNS, present-nonsingleton), background, and display size for the color (left panel) and intensity (right panel) conditions.

and display size (two and four letters). In the color condition, singleton value (i.e., red among green or green among red) was included as a between-subjects factor.

Color. The main effect of singleton value was not significant, nor did it interact with any other variables. All remaining factors produced significant main effects $[F(1,15)=6.10, p<.05$ for background; $F(2,30)=$ $29.26, p<.001$ for trial type; and $F(1,15)=145.26, p<$ .001 for display size]. There was also a significant trial type $\times$ display size interaction $[F(2,30)=13.77, p<$ $.001]$, indicating differences among the slopes of the three trial type functions. The three-way interaction between trial type, display size, and background, however, did not reach significance $(F<1)$. The lack of a threeway interaction confirms what is evident in the figure; the pattern of slopes was not influenced by the introduction of background dots.

Collapsed across background, the slopes for presentsingleton, present-nonsingleton, and absent conditions were $39.3,19.6$, and $48.5 \mathrm{msec}$, respectively. The slope for the present-singleton condition was significantly steeper than the slope for the present-nonsingleton condition $[t(15)=3.07, p<.01]$. In addition, both of these slope values were significantly greater than zero $[t(15)=$ $7.71, p<.001$, and $t(15)=5.51, p<.001$, respectively].

Error rates averaged $4.1 \%$ and tended to either increase or remain relatively constant with increases in display size, suggesting that the response time data are not contaminated by speed-accuracy tradeoffs. An ANOVA of error rates yielded significant main effects of trial type $[F(2,30)=5.61, p<.01]$ and display size $[F(1,15)=6.89, p<.05]$. There was also a significant interaction between these two variables $[F(2,30)=9.67$, $p<.001]$, indicating that the effect of display size on error rates varied with trial type. Specifically, display size had the largest effect in the present-singleton condition, producing an error slope of $2.5 \%$ compared with $0.1 \%$ and $-0.3 \%$ for the present-nonsingleton and absent conditions, respectively. As with response time, the difference in error slopes for the two present functions was significant $[t(15)=2.86, p<.05]$. Variation in background produced no main effects, nor did it enter into any interactions.

Intensity. Main effects were again significant for both trial type and display size $[F(2,30)=52.73, p<$ .001 , and $F(1,15)=112.21, p<.001$, respectively]. These two factors also entered into a significant interaction $[F(2,30)=6.87, p<.01]$. The background factor failed to produce a main effect, and, as in the color condition, did not enter into any interactions. Collapsed across background, the slopes for the present-singleton, present-nonsingleton, and absent conditions were 27.8 , 31.5 , and $50.5 \mathrm{msec}$, respectively. The slopes for the present-singleton and present-nonsingleton conditions were both significantly greater than zero $[t(15)=4.29$, $p<.05$, and $t(15)=6.70, p=.05]$, but they did not differ from one another $[t(15)=0.61, p>.05]$.

Error rates averaged $4.7 \%$. Again, error rates tended to increase or remain constant with display size. An ANOVA of error rates yielded only a significant main effect of trial type $[F(2,30)=3.53, p<.05]$. Error slopes for the present-singleton, present-nonsingleton, and absent functions were $1.4 \%, 0.2 \%$, and $-0.1 \%$, respectively.

Table 1

Error Rates (Percent) by Discontinuity Type (Color, Intensity), Background, Trial Type, and Display Size (Two or Four Letters) in Experiment 1

\begin{tabular}{|c|c|c|c|c|c|c|}
\hline \multirow[b]{3}{*}{ Background } & \multicolumn{6}{|c|}{ Trial Type } \\
\hline & \multicolumn{2}{|c|}{$\begin{array}{l}\text { Present- } \\
\text { Singleton }\end{array}$} & \multicolumn{2}{|c|}{$\begin{array}{c}\text { Present- } \\
\text { Nonsingleton }\end{array}$} & \multicolumn{2}{|c|}{ Absent } \\
\hline & 2 Letters & 4 Letters & 2 Letters & 4 Letters & 2 Letters & 4 Letters \\
\hline \multicolumn{7}{|c|}{ Color } \\
\hline No texture & 2.7 & 9.2 & 3.1 & 4.3 & 2.8 & 2.1 \\
\hline Texture & 3.3 & 6.7 & 4.8 & 4.0 & 3.2 & 2.7 \\
\hline \multicolumn{7}{|c|}{ Intensity } \\
\hline No texture & 3.3 & 6.7 & 4.5 & 5.6 & 4.6 & 3.1 \\
\hline Texture & 3.8 & 5.8 & 5.6 & 5.7 & 3.4 & 4.4 \\
\hline
\end{tabular}




\section{Discussion}

The addition of background texture dots had virtually no effect on the efficiency of visual search. Significant display size effects were apparent for all trial types, and the magnitude of these effects (i.e., the slopes of the response time functions) did not vary across background conditions. Thus, these results provide no support for the hypothesis that locally defined discontinuities in color and brightness, at least those defined over the range used in the present experiment, produce attentional capture.

Although significant display size effects were observed for all trial types, the slope for present-singleton trials in the color condition was reliably larger than the slope for the present-nonsingleton condition, in both response time and error rate. ${ }^{3} \mathrm{~A}$ similar trend was apparent in the intensity condition. We suspect that this cost in performance for present-singleton trials reflects a strategic bias against searching the singleton location. As display size increases, the percentage of trials on which the target is the singleton necessarily decreases. Thus, subjects may adopt a strategy of avoiding the singleton location with larger display sizes, producing larger slopes and more "misses." To the extent that these effects reflect "top-down" strategic factors, they provide further evidence that color and intensity singletons were unable to produce stimulus-driven attentional capture.

\section{EXPERIMENT 2}

The texture elements used in Experiment 1 were spaced approximately $1^{\circ}$ of visual angle from one another-a distance within the $2^{\circ}$ range proposed by Sagi and Julesz (1987) as being critical for the preattentive detection of feature discontinuities. The texture elements used by Sagi and Julesz, however, were line segments measuring $1^{\circ}$ of visual angle in extent. As pointed out by Sagi and Julesz, it is possible that the critical spatial range for preattentive feature detection is not a discrete value, but is a continuous function of the size of the elements comprising the texture. Given that the texture elements used in Experiment 1 measured only $.23^{\circ}$ of visual angle in extent, perhaps the $1^{\circ}$ of separation was still beyond the critical spatial range for preattentive detection. Thus, in Experiment 2, performance in a texture condition identical to that used in Experiment 1 was compared with a condition in which the texture dots were spaced at a distance equal to the extent of a single dot (i.e., $.23^{\circ}$ of visual angle). In addition, three different display sizes (three, five, and seven letters) were run in each condition.

\section{Method}

Subjects. Sixteen Villanova University undergraduates volunteered to participate in two 1 -h sessions conducted over 2 days. Each received credit toward fulfillment of a course requirement. The subjects were randomly assigned to one of the two betweensubjects conditions described below. All the subjects were tested for normal or corrected-to-normal visual acuity and color vision using a Titmus II vision tester.
Apparatus. The apparatus was identical to that used in Experiment 1.

Stimuli. Letter stimuli and texture elements were identical in dimension to those used in Experiment 1. To accommodate the larger display size, three additional letters (C, L, and F) were added to the population of letters from which displays were created. Locations were defined by the same imaginary octagon that was used in Experiment 1. Letters and their location for any given trial were chosen randomly and without replacement.

The texture matrix in which the letters appeared remained constant in spatial extent at $10.9^{\circ} \times 10.9^{\circ}$ of visual angle, but varied with respect to element density. The "sparse" texture was the same as that used in Experiment 1, with a $9 \times 9$ array of dots separated by $.92^{\circ}$ of visual angle. The "dense" texture consisted of a $27 \times 27$ array of dots separated by $.23^{\circ}$ of visual angle.

Design. A mixed design with one between-subjects factor and three within-subjects factors was used. The between-subjects factor was discontinuity type (color, intensity). As in Experiment 1, displays in each of these conditions contained one singleton letter. For half of the subjects in the color condition, the singleton was a red letter (IBM Color 12) among green letters and texture elements (IBM Color 10). The other half of the subjects received the reverse color assignment. In the intensity condition, all display items were white, but for half of the subjects one item was brighter (IBM Color 15; approximately $40 \mathrm{~cd} / \mathrm{m}^{2}$ ) than the rest (IBM Color 7; approximately $10 \mathrm{~cd} / \mathrm{m}^{2}$ ). The other half of the subjects received the reverse intensity assignment.

The three within-subjects factors were texture density (sparse, dense), display size (three, five, and seven letters), and trial type (present-singleton, present-nonsingleton, and absent). The target was present on half of the total trials and absent on the other half. When the target was present, it was the singleton on one third of the three letter displays, one fifth of the five letter displays, and one seventh of the seven letter displays.

The texture density factor was manipulated across two separate sessions for each subject. All other within-subjects variables were randomized within each block of trials. Each block contained slightly fewer Display Size 7 trials (28) than Display Size 3 or Display Size 5 trials ( 30 for each), due to the necessary constraints regarding the distribution of present-singleton and presentnonsingleton trials (see Jonides \& Yantis, 1988). Each session consisted of five blocks of 88 trials.

Procedure. The experiment consisted of two 60-min sessions on separate days. The subjects were randomly assigned to one of the two discontinuity type conditions and completed one session of the assigned condition with a sparse texture matrix and the other session with a dense texture matrix. The order of the texture density conditions was counterbalanced across subjects within each discontinuity type condition.

Each trial began with the presentation of the target letter to be searched for on that trial. The target appeared in the center of the screen, embedded in the texture matrix. In both discontinuity type conditions the target letter was white (IBM Color 7; approximately $25 \mathrm{~cd} / \mathrm{m}^{2}$, and the texture matrix was the color of the nonsingleton characters for that condition. After $1,000 \mathrm{msec}$, the target letter was removed and the texture matrix remained on the screen for $250 \mathrm{msec}$. The letters to be searched then appeared within the texture matrix and remained on the screen until a response was made. At that time, the letters were removed from the texture matrix and replaced with texture elements. The target for the next trial appeared after a $1,500-\mathrm{msec}$ ITI. In all other respects, the procedure was identical to that used in Experiment 1.

\section{Results}

Mean response times for each discontinuity type at each level of texture density, trial type, and display size 
COLOR

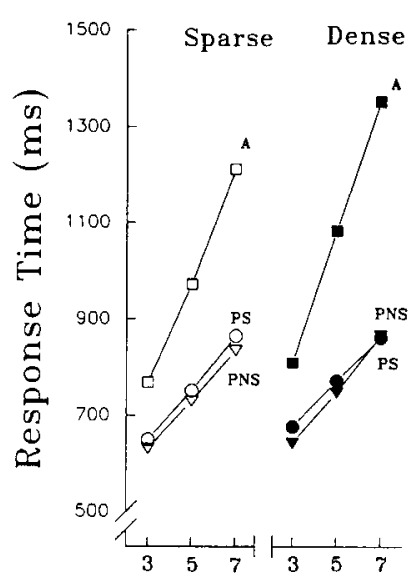

\section{INTENSITY}

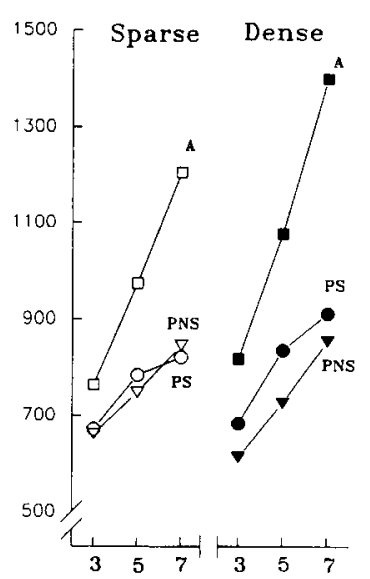

Display Size

Figure 3. Average mean response times as a function of trial type (A, absent; PS, present-singleton; PNS, present-nonsingleton), texture density, and display size for the color (left panel) and intensity (right panel) conditions.

are plotted in Figure 3, and corresponding error rates are listed in Table 2. Separate repeated measures ANOVAs were performed on the response time data from each of the discontinuity conditions. Each analysis included trial type (present-singleton, present-nonsingleton, and absent), display size (three, five, and seven letters), and texture density (sparse, dense). ${ }^{4}$

Color. Main effects were produced by trial type $[F(2,14)=109.33, p<.0001]$ and display size $[F(2,14)=150.39, p<.0001]$, and these two variables entered into a significant interaction $[F(4,28)=16.20$, $p<.0001]$. As is clear in the left panel of Figure 2, the pattern of slopes for the display-size/response-time functions across trial types was not influenced by texture density; the three-way interaction was not significant $[F(4,28)=1.69, p>.05]$.

Collapsed across the sparse and dense conditions, the slopes of the present-singleton, present-nonsingleton, and absent functions were 50,54 , and $123 \mathrm{msec}$, re-

spectively. The slope of the present-singleton function did not differ significantly from the slope of the presentnonsingleton condition $[t(7)=0.3, p>.05]$, providing no evidence of attentional capture.

The only significant effect of texture density was an interaction with trial type $[F(2,14)=9.47, p<.01]$. Simple effects analyses revealed that texture density had no effect on present-singleton or present-nonsingleton trials, but it did have a significant effect on absent trials. Dense textures produced longer response times than sparse textures $[t(7)=2.31, p<.05$, one-tailed].

Error rates for the color condition averaged $5.8 \%$. An ANOVA of error rates yielded significant main effects of background $[F(1,7)=22.98, p<.01]$, trial type $[F(2,14)=8.73, p<.01]$, and display size $[F(2,14)=$ $11.53, p<.01]$. The only significant interaction was between trial type and display size $[F(4,28)=3.67, p<$ $.05]$. Collapsed across background, the error slopes for the present-singleton, present-nonsingleton, and absent conditions were $2.7 \%, .02 \%$, and $1.1 \%$, respectively. As in Experiment 1, the error slope for the present-singleton condition was reliably steeper than the slope for the present-nonsingleton condition $[t(7)=2.8, p<.05]$, suggesting that there was again a bias against searching the display element at the singleton location.

Intensity. The analysis yielded main effects of trial type $[F(2,14)=52.80, p<.0001]$ and display size $[F(2,14)=158.94, p<.0001]$, as well as a significant interaction between these variables $[F(4,28)=28.75$, $p<.0001]$. Consistent with the color condition, the right panel of Figure 3 shows that texture density had little effect on the pattern of slopes for the display-size/ response-time functions across trial types. The texture density $\times$ trial type $\times$ display size interaction was once again not significant $[F(4,28)=0.90, p>.05]$.

Collapsed across the sparse and dense conditions, the slopes of the present-singleton, present-nonsingleton, and absent functions were 47,53 , and $128 \mathrm{msec}$, respectively. The slopes for the two present functions did not differ significantly $[t(7)=0.60, p>.05]$. Thus, there is no evidence that attention was captured by the intensity discontinuity.

Texture density entered into a marginal interaction with trial type $[F(2,14)=3.63, p<.06]$ and a reliable

Table 2

Error Rates (Percent) by Discontinuity Type (Color, Intensity), Texture Density, Trial Type, and Display Size (Three, Five, or Seven Letters) in Experiment 2

\begin{tabular}{|c|c|c|c|c|c|c|c|c|c|}
\hline \multirow{3}{*}{$\begin{array}{l}\text { Texture } \\
\text { Density }\end{array}$} & \multicolumn{9}{|c|}{ Trial Type } \\
\hline & \multicolumn{3}{|c|}{$\begin{array}{l}\text { Present- } \\
\text { Singleton }\end{array}$} & \multicolumn{3}{|c|}{$\begin{array}{c}\text { Present- } \\
\text { Nonsingleton }\end{array}$} & \multicolumn{3}{|c|}{ Absent } \\
\hline & 3 Letters & 5 Letters & 7 Letters & 3 Letters & 5 Letters & 7 Letters & 3 Letters & 5 Letters & 7 Letters \\
\hline \multicolumn{10}{|c|}{ Color } \\
\hline Sparse & 2.0 & 10.0 & 12.5 & 3.0 & 2.7 & 4.0 & 1.5 & 3.3 & 5.5 \\
\hline Dense & 8.0 & 6.7 & 18.8 & 5.8 & 3.5 & 5.0 & 2.3 & 1.8 & 7.1 \\
\hline \multicolumn{10}{|c|}{ Intensity } \\
\hline Sparse & 2.0 & 9.2 & 12.5 & 3.0 & 2.5 & 3.5 & 2.0 & 3.3 & 5.7 \\
\hline Dense & 7.0 & 8.3 & 11.2 & 3.5 & 4.0 & 4.8 & 2.7 & 3.0 & 8.4 \\
\hline
\end{tabular}


interaction with display size $[F(2,14)=12.29, p<$ $.001]$. Post hoc analyses established that the magnitude of the density effect was larger on absent trials than on present-nonsingleton trials $[t(7)=3.92, p<.05]$ and was larger for Display Size 7 than for Display Size 5 $[t(7)=3.41, p<.05]$ or Display Size $3[t(7)=4.25, p<$ $.05]$.

Error rates for the intensity condition averaged 5.4\%. An ANOVA of error rates yielded significant main effects of trial type $[F(2,14)=10.81, p<.01]$ and display size $[F(2,14)=6.50, p<.05]$; the largest number of errors occurred in the present-singleton condition with a display size of 7. No other main effects or interactions were significant.

\section{Discussion}

The data from the two discontinuity type conditions are remarkably consistent. In both conditions, the slopes of the present-singleton and present-nonsingleton functions did not differ significantly from one another and were approximately half the slope of the absent functions. More importantly, this pattern was obtained regardless of the density of the texture matrix. Thus, the results suggest that even when feature discontinuities are defined over a very small spatial range, subjects engage in a serial, self-terminating search of the display that does not automatically begin at the location of the discontinuity. In short, there was no evidence that the feature discontinuities in any of the conditions produced attentional capture.

Although increasing texture density did not appear to elicit involuntary attention shifts, it did have clear (negative) effects on search performance. Specifically, the interactions of texture density with both display size and trial type indicate that the dense texture lengthened response time for displays requiring a relatively extensive or exhaustive search. Such effects suggest that dense textures impair some iterative process involved in search, in that the cumulative impairment increases with the number of characters searched. One obvious possibility is that dense textures tend to mask the display characters, lengthening the identification time for each character searched.

\section{EXPERIMENT 3}

In the first two experiments there was no evidence of attentional capture by feature singletons, regardless of background condition. There are two alternative interpretations that must be ruled out, however, before concluding that the spatial range over which singletons are defined has no effect on their ability to capture attention. First, it is possible that the color and intensity singletons used in Experiments 1 and 2 were not detected preattentively, and that they were therefore unable to support attentional capture. Second, it is possible that the display elements were segregated from the background texture at a very early stage of processing, and therefore they had little or no effect on singleton processing. Under this scenario, singleton display elements might be defined only with respect to the other display elements, and not with respect to the density of the background texture. In other words, it is possible that the addition of background textures had no effect at all on the detection of color and intensity singletons, let alone their ability to capture attention.

To rule out these alternative interpretations, the subjects in Experiment 3 were shown displays similar to those used in the first two experiments, but were asked to simply indicate if a singleton in color or intensity was present or not, without regard to the identity of the singleton letter. If, as assumed in Experiments 1 and 2, the detection of singletons is (1) preattentive and (2) influenced by background texture density, then on present trials, response times should remain constant with changes in display size but should decrease as background density increases.

\section{Method}

Subjects. Twenty-four Villanova University undergraduates volunteered to participate in a single $1-\mathrm{h}$ session. Each received credit toward fulfillment of a course requirement. Half of the subjects were randomly assigned to the color condition, and half were assigned to the intensity condition. All the subjects were tested for normal or corrected-to-normal visual acuity and color vision using a Titmus II vision tester.

Apparatus. The apparatus was identical to that used in Experiment 1.

Stimuli. Letter stimuli and texture elements were identical in dimension and identity to those used in Experiment 2. Letters and their location for any given trial were chosen randomly and without replacement.

Design. A mixed design with one between-subjects factor and three within-subjects factors was used. The between-subjects factor was discontinuity type (color, intensity). For half of the displays in each condition exactly one singleton letter was present, and for the other half all displays elements (including background texture) were uniform in color or intensity. In the color condition, the singleton was a red letter (IBM Color 12) among green letters and green texture elements (IBM Color 10). In the intensity condition, the singleton was brighter (IBM Color 15; approximately $40 \mathrm{~cd} / \mathrm{m}^{2}$ ) than the rest of the display elements (IBM Color 7; approximately $10 \mathrm{~cd} / \mathrm{m}^{2}$ ). On present trials, singleton location was chosen randomly.

The three within-subjects factors were texture density (no texture, sparse texture, dense texture), display size (three, five, and seven letters), and trial type (present, absent). The texture density factor was manipulated across blocks of trials and the order of texture conditions was balanced across subjects. All other withinsubjects variables were randomized within each block of trials. Within each texture condition, there were three blocks of 36 trials. Within each block, each display size occurred equally often.

Procedure. Each trial began with the presentation of a fixation cross in the center of the screen, appearing either alone (in the notexture condition) or embedded in a texture matrix (in the sparse and dense texture conditions). After $1,000 \mathrm{msec}$, the fixation cross was removed for $250 \mathrm{msec}$. This was followed by the presentation of the display letters, which remained on the screen until a response was made. At that time, the letters disappeared and were replaced with texture elements in the sparse and dense texture conditions. The fixation cross for the next trial appeared after a 1,500 -msec ITI. 
The subjects were instructed to determine whether a singleton letter was present, regardless of the identity of the letter. In all other respects, the procedure was identical to that used in Experiment 2.

\section{Results}

Mean response times for each discontinuity type at each level of texture density, trial type, and display size are plotted in Figure 4, and corresponding error rates are listed in Table 3. Separate repeated measures ANOVAs were performed on the response time data from each of the discontinuity conditions. Each analysis included trial type (present, absent), display size (three, five, and seven letters), and texture density (no texture, sparse texture, dense texture).

Color. The effect of trial type was significant; absent trials produced longer response times than present trials $[F(1,11)=12.33, p<.01]$. Response time also varied with display size $[F(2,22)=4.17, p<.05]$. Tukey's post hoc tests $(\alpha=.05)$ revealed that response times for Display Size 5 were significantly faster than those for Display Size 3. The difference between mean response times for Display Sizes 3 and 7 and 5 and 7 were not significant. For present trials, the slopes for the display size factor were $-3.25,-3.25$, and $0 \mathrm{msec}$ for the no-texture, sparse texture, and dense texture conditions, respectively. The corresponding values for absent trials were $-5.0,-7.5$, and $5.75 \mathrm{msec}$.

The only significant interaction was between background and trial type $[F(2,22)=3.93, p<.05]$. Simple effects analyses established that background had a significant effect on present trials $[F(2,22)=3.88, p<$ $.05]$, but no effect on absent trials $(F<1)$. Tukey's post hoc analyses of present trials established that the mean response time for the dense texture condition was reliably faster than those for the sparse texture or no-texture conditions. The latter two means did not differ.

Error rates for the color condition were very low, averaging just over 1\%. An ANOVA of error rates with trial type, display size, and background as factors yielded no significant effects.

Intensity. As in the color condition, trial type produced a significant main effect $[F(1,11)=19.91, p<$
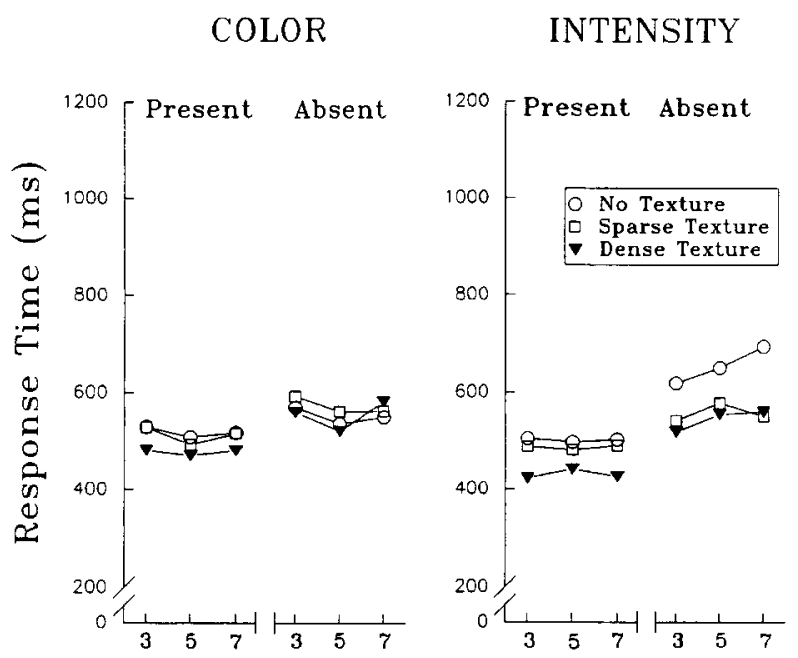

Display Size

Figure 4. Average mean response times as a function of trial type, background, and display size for the color (left panel) and intensity (right panel) conditions.

$.01]$. Background produced the only other significant effect $[F(2,22)=9.33, p<.01]$. Tukey's tests $(\alpha=.05)$ established that response times for the no-texture condition were reliably longer than times for the sparse and dense texture conditions. The latter means did not differ reliably. The slopes for the display size factor on present trials were $0.25,0.25$, and $-0.5 \mathrm{msec}$ for the no-texture, sparse texture, and dense texture conditions, respectively. The corresponding values for absent trials were $2.25,10.5$, and $18.75 \mathrm{msec}$.

Planned comparisons were also conducted to determine the effects of background at each level of trial type. For both present and absent trials, response time decreased reliably as background texture increased $[F(2,22)=25.21, p<.001 ; F(2,22)=4.62, p<.05$, respectively]. For present trials, Tukey's tests revealed significant differences among the means for all the background conditions. For absent trials, the mean for the no-texture condition differed reliably from the means

Table 3

Error Rates (Percent) by Discontinuity Type (Color, Intensity), Background, Trial Type, and Display Size (Three, Five, or Seven Letters) in Experiment 3

\begin{tabular}{|c|c|c|c|c|c|c|}
\hline \multirow{3}{*}{$\begin{array}{l}\text { Background } \\
\text { Texture }\end{array}$} & \multicolumn{6}{|c|}{ Trial Type } \\
\hline & \multicolumn{3}{|c|}{ Present } & \multicolumn{3}{|c|}{ Absent } \\
\hline & 3 Letters & 5 Letters & 7 Letters & 3 Letters & 5 Letters & 7 Letters \\
\hline \multicolumn{7}{|c|}{ Color } \\
\hline None & 1.4 & 0.5 & 0.9 & 2.8 & 0.9 & 0.9 \\
\hline Sparse & 1.8 & 0.9 & 1.4 & 2.3 & 0.5 & 0.5 \\
\hline Dense & 2.3 & 1.4 & 1.4 & 0.5 & 1.8 & 0.9 \\
\hline \multicolumn{7}{|c|}{ Intensity } \\
\hline None & 1.8 & 0.4 & 0.9 & 2.8 & 0.4 & 1.4 \\
\hline Sparse & 0.5 & 2.3 & 2.3 & 1.4 & 2.3 & 0.5 \\
\hline Dense & 1.4 & 0.9 & 1.4 & 1.8 & 0.5 & 0.0 \\
\hline
\end{tabular}


for the sparse and dense texture conditions, but the latter two did not differ signficantly from one another.

Mean error rate for the intensity condition was just over $1 \%$. An ANOVA yielded a marginally significant interaction between background and display size $[F(4,44)=$ $2.73, p=.04]$. No other effects were significant.

\section{Discussion}

The results of this experiment provide clear evidence that the color and intensity singletons used in the first two experiments were detected preattentively. Specifically, the time to detect the presence of a color or intensity singleton was relatively unaffected by display size, as evidenced by the shallow slopes in all conditions. Indeed, the only reliable effect of display size was an overall reduction in response time for Display Size 5 in the color condition, for which we have no particular explanation at present. In no condition was response time for Display Size 7 reliably different from Display Size 3.

The results also provide evidence that is inconsistent with the notion that singletons are processed independently of background texture. Specifically, color and intensity singletons were detected more rapidly when presented in the context of a dense texture than when presented alone or in a sparse texture. The effect of background density on absent trials was more variable; background produced no effect in the color condition, and response times for sparse and dense textures did not differ in the intensity condition. Although we had no a priori predictions concerning the effect of background on absent trials, we suspect that this variability may reflect the influence of decision-level effects, such as variations in response "deadlines."

In sum, this experiment suggests that the background density manipulations used in Experiments 1 and 2 were indeed effective in enhancing the preattentive detectability of color and intensity singletons.

\section{GENERAL DISCUSSION}

The present experiments were conducted to determine whether the ability of feature discontinuities to capture attention is influenced by the spatial range over which the discontinuities are defined. Given the results of previous experiments on the effect of display density on visual search and texture segregation (e.g., Sagi \& Julesz, 1987), it was hypothesized that locally defined feature discontinuities would indeed produce attentional capture. In Experiment 1, locally defined discontinuities in color and intensity were created by embedding display characters in a dot texture. Regardless of whether or not the discontinuities were defined locally (i.e., whether or not the characters were embedded in a texture), the effect of display size was virtually unaffected by the singleton status of the target. Experiment 2 confirmed that this pattern does not change as the density of the textured background increases. Experiment 3 established that the background density manipulation does indeed influence the preattentive detectability of color and intensity singletons. Thus, the results of these experiments establish that the spatial region over which feature singletons are defined can influence their detection, but it has no impact on their ability to produce attentional capture, at least as measured by the paradigm used here.

These results have several implications for models of visual search and selective attention. First, they suggest that the display density effects observed in previous visual search and texture discrimination studies (as well as in the present Experiment 3) probably reflect more efficient operation of voluntary guidance strategies, rather than the stimulus-driven capture of attention by locally defined discontinuities. This interpretation is consistent with the work of Bacon and Egeth (1991), who found that density effects in visual feature search are restricted to the spacing between homogeneous nontargets rather than between nontargets and targets. These authors argue that increasing display density results in more efficient perceptual grouping of nontargets, increasing the ease with which they are rejected from search. Similar notions have been proposed by others (e.g., Duncan \& Humphreys, 1989; Wolfe et al., 1989).

Second, the results provide evidence that is consistent with Jonides and Yantis's (1988) claim that attentional capture is elicited only by abrupt changes in luminance over time and not by static feature singletons. This conclusion must be accepted cautiously, however, in light of recent work in a slightly different experimental paradigm, which demonstrated that irrelevant feature discontinuities can, in fact, produce significant distraction effects indicative of attentional capture (Bacon \& Egeth, 1994; Folk et al., 1992, 1994; Pashler, 1988; Theeuwes, 1991, 1992). For example, both Pashler (1988, Experiment 6) and Theeuwes (1992) found that a color singleton interfered with search for a target defined by a form singleton. Furthermore, Folk et al. (1992), using a variant of the spatial cuing paradigm, found that a completely uninformative spatial cue defined by a discontinuity in color produces significant cuing effects for a subsequent target that is also defined by a color discontinuity.

How can the present results be reconciled with the results of these previous studies? Folk et al. (1992; Folk, Remington, \& Johnston, 1993) have recently argued that attentional capture can be produced by a variety of stimulus properties (including feature singletons), but that all capture is contingent on the establishment of internal attentional control settings. Stimuli with properties consistent with the current attentional control settings elicit attentional shifts that can violate the observer's intentions. Stimuli that are not consistent with the current attentional set will not produce capture. Thus, in Folk et al.'s (1992) study, evidence of attentional capture by a feature singleton was observed only when the subjects were "set" for a feature singleton. Likewise, the interference produced by color singletons when searching for form singletons in the studies by 
Pashler (1988) and Theeuwes $(1991,1992)$ can be accounted for by assuming that the subjects were "set" to respond to singletons in general, without regard to their defining dimensions (see Bacon \& Egeth, 1994).

With respect to the paradigm developed by Yantis and Jonides (1984) and used in the present experiments, Folk et al. $(1992,1993)$ argue that the presentation of the target display is signaled by dynamic changes in the luminance of the display items (all items are abruptly onset). Thus, the task may induce subjects to establish an attentional "set" for dynamic luminance change. Accordingly, the lack of evidence for capture by static discontinuities in the present experiments may simply reflect the influence of an attentional set for luminance change. This interpretation is by no means uncontroversial (see Yantis, 1993), but even if it is an accurate interpretation of the data, the present experiments establish that when feature discontinuities are defined over a small spatial range, they are unable to override existing attentional control settings.

Although the present results suggest that manipulating the spatial range over which feature singletons are defined does not affect their ability to produce attentional capture, the results do not rule out the possibility that other factors could render feature singletons salient enough to have significant effects on attentional allocation. In a recent study using Jonides and Yantis's (1988) paradigm, Todd and Kramer (in press) showed that, at large display sizes (i.e., 16-25 elements), a response time advantage does emerge for singleton targets relative to nonsingleton targets, even when interelement distance is held constant. The authors argue that, at larger display sizes, the salience of feature singletons is enhanced to the point that they can produce attentional "misguidance." Thus, their results suggest that there may in fact be circumstances under which the bottomup salience of feature singletons can have overriding influence on the guidance of spatial attention.

\section{Conclusions}

Attention allocation in visual search is clearly a complex phenomenon involving top-down, goal-directed processes as well as bottom-up, stimulus-driven processes. The present study establishes that, with respect to stimulus-driven attention allocation, the spatial range over which feature discontinuities in color and intensity are defined has no influence on their ability to capture attention or to override existing attentional control settings. The results also suggest that the density effects observed in previous studies of feature search are not the result of attentional capture by feature discontinuities, but instead reflect the enhancement of voluntary guidance mechanisms.

\section{REFERENCES}

BACON, W., \& EGETH, H. (1991). Local processes in preattentive feature detection. Journal of Experimental Psychology: Human Perception \& Performance, 17, 77-90.
BaCon, W., \& Egeth, H. (1994). Overriding stimulus-driven attentional capture. Perception \& Psychophysics, 55, 485-496.

Beck, J., Prazdny, K., \& Rosenfeld, A. (1983). A theory of textural segmentation. In J. Beck, B. Hope, \& A. Rosenfeld (Eds.), Human and machine vision (pp. 1-38). London: Academic Press.

Bergen, J. R., \& Julesz, B. (1983). Parallel versus serial processing in rapid pattern detection. Nature, 303, 696-698.

Bravo, M. J., \& Nakayama, K. (1992). The role of attention in different visual-search tasks. Perception \& Psychophysics, 51, $465-$ 472.

CAVE, K. R., \& WolfE, J. M. (1990). Modelling the role of parallel processing in visual search. Cognitive Psychology, 22, 225-271.

CoNNOR, J. M. (1972). Effects of increased processing load on parallel processing of visual displays. Perception \& Psychophysics, 12, 121-128.

DunCan, J., \& Humphreys, G. W. (1989). Visual search and stimulus similarity. Psychological Review, 96, 433-458.

EGETH, H., JoNIDES, J., \& WALL, S. (1972). Parallel processing of multielement displays. Cognitive Psychology, 3, 647-698.

Folk, C. L., \& EgETH, H. (1989). Does the identification of simple features require serial processing? Journal of Experimental Psychology: Human Perception \& Performance, 15, 97-110.

Folk, C. L., Remington, R. W., \& Johnston, J. C. (1992). Involuntary covert orienting is contingent on attentional control settings. Journal of Experimental Psychology: Human Perception \& Performance, 18, 1030-1044.

Folk, C. L., Remington, R. W., \& Johnston, J. C. (1993). Contingent attentional capture: A reply to Yantis (1993). Journal of Experimental Psychology: Human Perception \& Performance, 19, 682685.

Folk, C. L., Remington, R. W., \& Wright, J. H. (1994). The structure of attentional control: Contingent attentional capture by apparent motion, abrupt onset, and color. Journal of Experimental Psychology: Human Perception \& Performance, 20, 317-329.

Jonides, J., \& YANTIS, S. (1988). Uniqueness of abrupt visual onset in capturing attention. Perception \& Psychophysics, 43, 346-354.

Julesz, B. (1986). Texton gradients: The texton theory revisited. Biological Cybernetics, 54, 245-251.

Kahneman, D., \& Henik, A. (1981). Perceptual organization and attention. In M. Kubovy \& J. R. Pomerantz (Eds.), Perceptual organization (pp. 181-211). Hillsdale, NJ: Erlbaum.

Косн, C., \& Ullman, S. (1985). Shifts in selective visual attention: Toward the underlying neural circuitry. Human Neurobiology, 4, 219-227.

NEISSER, U. (1967). Cognitive psychology. New York: AppletonCentury-Crofts.

NoTHDURFT, H.-C. (1985). Sensitivity for structure gradient in texture discrimination tasks. Vision Research, 25, 1957-1968.

NothDURFT, H.-C. (1992). Feature analysis and the role of similarity in preattentive vision. Perception \& Psychophysics, 52, 355-375.

PASHLER, H. (1988). Cross-dimensional interaction and texture segregation. Perception \& Psychophysics, 43, 307-318.

SAGI, D., \& JuLESZ, B. (1987). Short-range limitation on detection of feature differences. Spatial Vision, 2, 39-49.

TheEuwes, J. (1990). Perceptual selectivity is task-dependent: Evidence from selective search. Acta Psychologica, 74, 81-99.

Theeuwes, J. (1991). Cross-dimensional perceptual selectivity. Perception \& Psychophysics, 50, 184-193.

Theeuwes, J. (1992). Perceptual selectivity for color and form. Perception \& Psychophysics, 51, 599-606.

TodD, S., \& Kramer, A. (in press). Attentional misguidance in visual search. Perception \& Psychophysics.

Treisman, A., \& Gelade, G. (1980). A feature integration theory of attention. Cognitive Psychology, 12, 97-136.

Treisman, A., \& Sourher, J. (1985). Search asymmetry: A diagnostic for preattentive processing of separable features. Journal of Experimental Psychology: General, 114, 285-310.

Ullman, S. (1984). Visual routines. Cognition, 18, 97-159.

WOLFE, J. (1992, November). Guided search 2.0: A revised model of visual search. Paper presented at the meeting of the Psychonomic Society, St. Louis, MO. 
Wolfe, J. M., Cave, K. R., \& Franzel, S. L. (1989). Guided search: An alternative to the feature integration model for visual search. Journal of Experimental Psychology: Human Perception \& Performance, 15, 419-433.

YANTIS, S. (1993). Stimulus-driven attentional capture and attentional control settings. Journal of Experimental Psychology: Human Perception \& Performance, 19, 676-681.

YANTIS, S., \& Hillstrom, A. P. (1994). Stimulus-driven attentional capture: Evidence from equiluminant visual objects. Journal of Experimental Psychology: Human Perception \& Performance, 20, 95 107.

YanTis, S., \& Johnson, D. N. (1990). Mechanisms of attentional priority. Journal of Experimental Psychology: Human Perception \& Performance, 16, 812-825.

YANTIS, S., \& JoNES, E. (1991). Mechanisms of attentional selection: Temporally modulated priority tags. Perception \& Psychophysics, 50, 166-178.

YANTIS, S., \& JonidES, J. (1984). Abrupt visual onsets and selective attention: Evidence from visual search. Journal of Experimental Psychology: Human Perception \& Performance, 10, 601-621.

YANTIS, S., \& JonIDES, J. (1990). Abrupt visual onsets and selective attention: Voluntary versus automatic allocation. Journal of Experimental Psychology: Human Perception \& Performance, 16, 121-134.

\section{NOTES}

1. Recent work by Yantis and Hillstrom (1994) suggests that it is the abrupt onset of a new "object" rather than abrupt onset per se that produces attentional capture.
2. One might question why the same argument would not hold for abrupt onsets, which produced attentional capture even at the relatively large interelement distances used by Jonides and Yantis (1988). Unlike feature singletons, whose existence (and presumably coding) is dependent on the presence of other display elements, abrupt onsets exist (and are presumably coded) independently of the presence of other display elements. Thus, there is little reason to expect interelement distance to affect capture by onsets.

3. A similar effect on error rates is apparent for color singletons in Jonides and Yantis (1988, Experiment 2).

4. Preliminary analyses were conducted for each condition to determine the influence of discontinuity value (red/green, bright/dim) on the pattern of results. In the color condition there was no main effect of discontinuity value, nor did this variable enter into any interactions (all $p s>.05$ ). In the intensity condition, discontinuity type (bright/ dim) produced a significant main effect $[F(1,6)=6.05, p<.05]$ and entered into a two-way interaction with trial type $[F(2,12)=13.68$, $p<.05]$ as well as a three-way interaction with background density and trial type $[F(2,12)=4.36, p<.05]$. These effects are most likely the result of the difference in salience between bright and dim characters. It is beyond the scope of the present paper, however, to attempt to provide a complete account of these effects. The important point is that discontinuity value did not enter into a four-way interaction with trial type, display size, and background density. This indicates that the pattern of results shown in the right panel of Figure 3 is consistent across both dim and bright discontinuities.

(Manuscript received June 28, 1993; revision accepted for publication February 14, 1994.) 